\title{
Complex atypical hyperplasia: Are we pushing the limits?
}

\author{
Michelle F BENOIT* \\ Gynecologic Oncology, Kaiser Permanente Washington, USA
}

\begin{abstract}
Background: This review was initiated to identify the number of patients who underwent hysterectomy for complex atypical hyperplasia $(\mathrm{CAH})$ who then needed further surgical staging due to pathologic identification of an invasive uterine cancer. We then evaluated the need for secondary surgery using two different normograms for uterine cancer lymph node dissection.

Materials and methods: We reviewed all hysterectomy surgeries performed at two of four sites of a Washington state medical group. We captured all patients with a preoperative diagnosis of $\mathrm{CAH}$. We reviewed their preoperative endometrial sampling pathology and their final surgical hysterectomy pathology. We abstracted additional data to include age, BMI, ethnicity, comorbidities of DM and HTN, preoperative sampling methods, and definitive surgical approach. We then identified the number of those patients who would need further surgical staging using two validated uterine cancer staging normograms.

Results: 1515 total hysterectomy procedures were reviewed between 2013 and 2017. 78 patients with were identified with a preoperative diagnosis of CAH. 60 hysterectomies were done by gynecologic oncologists, and 18 by general gynecology. 51 uterine cancers were found. Of these, 33 would qualify for further surgical staging per Mayo criteria and 5 would qualify per a French uterine cancer normogram.

Discussion: $65 \%$ of all CAH were found to have uterine cancer at final surgical pathology. 64.1\% qualified for further surgical staging per Mayo Criteria and 9.8\% by the French normograms. When size criteria were removed from the Mayo criteria, $7.8 \%$ would have qualified for staging. No surgeon (general gynecology vs subspecialty) performing hysterectomy adversely affected surgical or oncologic outcomes in this population of women with a preoperative diagnosis of CAH/EIN. EIN may be the preferred nomenclature as it can risk stratify patients for better classification of disease and is the recommended international pathology terminology.
\end{abstract}

\section{Introduction}

Background: Recamier, in 1850 , was the first to recognize the condition of endometrial hyperplasia Endometrial hyperplasia is usually the result of a persistent, prolonged, estrogenic stimulation of the endometrium either from obesity, exogenous estrogens, prolonged anovulatory cycles, with or without genetic stimuli [1]. Almost 67 years ago, Hertig and Sommers, proposed the theory of progression of endometrial hyperplasia to adenocarcinoma, through a stage of atypical changes.

The World Health Organization (WHO) in 1994 separated endometrial hyperplasia into 4 categories, of simple and complex hyperplasia with or without cellular atypia [2], based on Kurman et al's terminology. These categories are descriptive in nature, and interpretation is subjective; accordingly, studies indicate poor reproducibility of the individual case classification. Moreover, the individual categories do not suggest specific management algorithms.

Uterine pre-cancer terminology has recently undergone descriptive changes to clearly distinguish between clinicopathologic entities that are managed differently, and to highlight the necessity of definitive management of precancerous states. The older terminology of complex hyperplasia and complex atypical hyperplasia (CAH), denoted by Kurman et al, has now been grouped together and designated "endometrial intraepithelial neoplasia" (EIN) by ACOG and SGO. This new categorization can then more effectively guide the clinician in management of the patient with such diagnoses. Most commonly, surgical management has been prescribed for the precancerous EIN lesions. In lower risk lesions (previously defined as $\mathrm{SH}$ or $\mathrm{CH}$ without atypia) or unsuitable surgical candidates, progestin therapies, such as a hormonal IUD or oral systemic hormones, are recommended [3].

\section{Endometrial hyperplasia classification systems}

In the endometrial hyperplasia schema, pathologic criteria were used to develop three disease categories: 1) benign (benign endometrial hyperplasia), 2) premalignant (endometrial intraepithelial neoplasia/ EIN), and 3) malignant (endometrial adenocarcinoma, endometrioid type, well differentiated) (Tables 1 and 2).

A histologic comparison study (GOG 167) published in 2006 reviewed 289 specimens with a preoperative diagnosis of CAH. A centralized review with a panel of gynecologic pathologists rendered $42.6 \%$ of specimens with an initial preoperative diagnosis of $\mathrm{CAH}$ to have a concurrent endometrial carcinoma (123 of 289 specimens) at final hysterectomy. Of these, $30.9 \%$ (38 of 123 specimens) were myoinvasive, and 10.6\% (13 of 123 specimens) involved the outer $50 \%$ of the myometrium. There was a high rate of inter-observer discrepancy between specimens: 74 of 289 specimens (25.6\%) were diagnosed as less than atypical endometrial hyperplasia, 115 of 289 specimens (39.8\%) were diagnosed as $\mathrm{CAH}$, and 84 of 289 specimens (29.1\%) were diagnosed as endometrial carcinoma. In 5.5\% (16 of 289 specimens), there was no consensus on the biopsy diagnosis. Among the women who had hysterectomy specimens with carcinoma, 14 of 74 women $(18.9 \%)$ had a study panel biopsy consensus diagnosis of less than $\mathrm{AEH}, 45$ of 115 women (39.1\%) had a study panel biopsy

*Correspondence to: Michelle F. BENOIT, M.D, Gynecologic Oncology, Kaiser Permanente Washington, 11511 NE 10th St. Bellevue, WA 98004, USA, Tel: 425-502-3000, 425-502-3459; E-mail: Michelle.F.Benoit@kp.org

Received: September 05, 2018; Accepted: September 17, 2018; Published: September 20, 2018 
Table 1. Diagnostic Criteria for Endometrial Intraepithelial Neoplasia*

\begin{tabular}{|l|l|l|l|}
\hline Nomenclature & Topography & Functional Category & Treatment \\
\hline Benign endometrial hyperplasia & Diffuse & Prolonged estrogen effect & Hormonal therapy, symptomatic \\
\hline Endometrial intraepithelial neoplasia & Focal progressing to diffuse & Precancerous & Hormonal therapy or surgery \\
\hline $\begin{array}{l}\text { Endometrial adenocarcinoma, endometrioid } \\
\text { type, well differentiated }\end{array}$ & Focal progressing to diffuse & Malignant & Surgery, stage based \\
\hline
\end{tabular}

*Prior terminology was atypical endometrial hyperplasia [4,5].

Table 2. Definitions of Endometrial Intraepithelial Neoplasia* Criteria

\begin{tabular}{|l|l|}
\hline Endometrial Intraepithelial Neoplasia* Criteria & Comments \\
\hline Architecture & Area of glands greater than stroma (volume percentage stroma less than 55\%) \\
\hline Cytology & Cytology differs between architecturally crowded focus and background \\
\hline Size greater than $1 \mathrm{~mm}$ & Maximum linear dimension exceeds 1 mm \\
\hline Exclude mimics & Benign conditions with overlapping criteria (ie, basalis, secretory, polyps, repair) \\
\hline Exclude cancer & Carcinoma if maze-like glands, solid areas, or appreciable cribriforming \\
\hline
\end{tabular}

*Prior terminology was atypical endometrial hyperplasia.

consensus diagnosis of $\mathrm{CAH}$, and 54 of 84 women (64.3\%) had a study panel diagnosis of carcinoma. Among women who had no consensus in their biopsy diagnosis, 10 of 16 women (62.5\%) had carcinoma in their hysterectomy specimens [6].

The diagnosis of uterine cancer at the time of hysterectomy has been challenging as the question of lymph node involvement then follows. Comprehensive staging is recommended for certain subtypes and risk factors. Uterine cancers have been divided into two types: type I as the more prevalent endometrioid subtype, and type II tumors which tend to be the more aggressive subtypes to include the serous, clear cell, and carcinosarcoma histologies [7]. The transition from endometrial hyperplasia to uterine adenocarcinoma has most often been seen in type I tumors. Molecular analysis of uterine tumors is shedding further light into uterine cancer subgrouping but this is based on DNA and copy number analysis, not histologic appearance--which is the main method of pathologic diagnosis at this time [8].

Different normograms have been developed to help guide the gynecologist/gynecologic oncologist towards the necessity of surgical staging with lymph node dissection $[9,10,11]$. Lymph node dissection has not been found to be therapeutic but has been shown to be prognostic [12]. Its prognostic value then lies in its guidance for decisions regarding adjuvant therapies. These include targeted radiation fields, systemic chemotherapy, a combination of modalities, and evolving biologic and immunologic therapies.

Gynecologists have been the mainstay for surgical management of precursor lesions (CAH/EIN and less). As the subspecialty of gynecologic oncology evolved, the vigilance of gynecologists has grown and referrals to subspecialty have increased, including those for precancer lesions. This may be due to less surgical training as hysterectomy numbers from residency are much lower due to medical management of gynecologic diagnoses, perhaps from fear of litigation, possible concern for patients needing second surgical staging procedures, and otherwise. The question of the necessity of referral for $\mathrm{CAH}$ to the subspecialist has then arisen, with data from GOG 167 providing much of the drive.

We then wanted to review the numbers of CAH identified within our medical group. We wanted to review the concordant rate of uterine cancer diagnosed on final pathology. But we ultimately wanted to identify how many of those patients would have needed a second staging surgery defined by well-known normograms. We wanted to ensure we were not compromising patient outcomes at primary surgery when gynecology, not subspecialty, performed the hysterectomy.

\section{Materials and methods}

We reviewed all patients who underwent hysterectomy at two of four sites at a statewide HMO in Washington State from January 2013-March 2018. Patients were identified from the electronic medical records for procedure and preoperative diagnosis. Demographics were abstracted to include age, ethnicity, comorbidities of DM, HTN, and BMI. Preoperative endometrial sampling type was reviewed (office biopsy, D\&C, hysteroscopy). Pathology was reviewed to include preoperative and final surgical results. Grade, histology, tumor size, depth of invasion, and LVSI were included for review. The numbers of patients who were advised to undergo or who did undergo second surgical staging was also documented. Final stage was then assigned where appropriate. The Mayo and French normograms were used to stratify risk for needing surgical LN staging with their pre-weighted risks of known LN metastasis available for review. Graphpad and Sociscistatistics were used for statistical analysis and a $\mathrm{p}$ value of $<0.5$ was considered significant. IRB approval was obtained.

\section{Results}

One thousand-five-hundred-fifteen patients who underwent hysterectomy were identified from 2014-2017. Pathology was reviewed on all patients. Two patients with a final diagnosis of uterine cancer were removed from the cohort as there was no preoperative endometrial sampling. One patient, who had an endometrial biopsy showing EIC/ serous uterine cancer was resampled and the second biopsy "reverted" to $\mathrm{CAH}$, was also removed due to her discordant cancer diagnosis. Seventy-eight hysterectomies for CAH/EIN were performed. All patients had a preoperative diagnosis of CAH/EIN. Twenty-seven patients had a final diagnosis of CAH/EIN or less, and 51 patients had a final surgical pathologic diagnosis of uterine cancer.

Ethnicity was reviewed. There were 6 African American, 7 Asian, 4 Hispanic, and 61 Caucasian patients. Those with final pathology consisting of $\mathrm{CAH}$ were 21 Caucasian, 1 Asian, 1 Hispanic, and 1 African American. The average age of the entire cohort was 58 years. The average age of the CAH/EIN group was 57.51 (range 40-70) and the average age of the cancer group was 58.5 (range 26-83); the difference between cohort ages was not found to be different (unpaired $\mathrm{t}$ test $\mathrm{p}=$ 0.6654 ; $95 \%$ CI -3.77 to 5.87 ).

BMI was also calculated for each patient trying to associate higher BMI with more estrogen production, possibly tipping the scale towards a diagnosis of cancer. The average BMI for the CAH/EIN group was 40.08 and the average BMI of the cancer group was 38.54; and was not 
found to be different (unpaired t test $\mathrm{p}=0.5059 ; 95 \% \mathrm{CI}:-6.1$ to $3.04, \mathrm{t}$ $=0.6683$ ). Thirty-one total patients had a diagnosis of DM. There were 10 in the CAH/EIN cohort, and 21 in the cancer cohort. There was no difference between groups (unpaired t two-tailed $\mathrm{p}=0.6766$; 95\% CI: -0.19 to $0.29, \mathrm{t}=0.4187$ )

Fifty-two patients total carried a diagnosis for HTN. There were 15 in the CAH/EIN group and 37 in the cancer group. When the two groups were compared there was no significant difference found (unpaired $\mathrm{t}$ test two-tailed $\mathrm{p}=0.1016 ; 95 \% \mathrm{CI}$ : -0.04 to $0.4, \mathrm{t}=1.6573$ ). Endometrial Stripe (EMS) thickness was compared between groups. The average total EMS thickness was $1.80 \mathrm{~cm}$. The average thickness for those in the CAH/EIN group was 1.97, and for those who had cancer on final diagnosis was 2.44. The difference between groups was not found to be significant (unpaired $\mathrm{t}$ test two-tailed $\mathrm{p}=0.4127 ; 95 \% \mathrm{CI}$ : -0.9113 to $2.1933, \mathrm{t}=0.8244$ ).

Sampling for preoperative diagnosis was reviewed to see if one method was better than another, or if 2 samples were better than just one sample. Fifty-nine patients had an office endometrial biopsy (EMB), of those, 18 were patients had a final diagnosis of CAH/EIN patients and 49 had a final diagnosis of cancer. Statistically there was no difference between EMB for those who had a final diagnosis of CAH/ EIN vs cancer (unpaired $t$ test two-tailed $\mathrm{p}=0.1327 ; 95 \% \mathrm{CI}$ : -0.05 to $0.35, t=1.5201$ ). Twenty-five patients had hysteroscopy D\&C. Twelve patients who had a D\&C were in the CAH/EIN cohort and 13 were in the cancer cohort. There was no statistically significant difference in finding cancer by sampling method (unpaired two tailed t-test $\mathrm{p}=0.8342$ 95\% CI; $0.23-0.43, \mathrm{t}=3.488$ ) Five patients in each group had 2 biopsies, first an EMB, followed by a hysteroscopy. There was no significance between groups (unpaired two tailed t-test $\mathrm{p}=0.3112 ; 95 \%$ CI; 0.25--0.08, $\mathrm{t}=1.0197)$.

Preoperative pathology was reviewed. Preoperative pathology ranged from: "complex atypical hyperplasia"; to "complex atypical hyperplasia cannot rule out low grade endometrial neoplasm"; and "at least complex atypical hyperplasia". Thirty-two patients had a preoperative diagnosis of "at least CAH". One patient with a final diagnosis of CAH/EIN had the preoperative diagnosis of "at least". We compared this to the 31 patients with a final pathologic diagnosis of uterine cancer and found this was significant (unpaired test two-tailed $\mathrm{p}=0.0005,95 \%$ CI: 0.18 to $0.62, \mathrm{t}=3.6669)$.

Regarding those found to have cancer on final diagnosis: there were 41 staged T1AG1, 3 with stage T1AG2, 4 with stage T1BG1, 1 stage T1BG2, 1 stage T2, and 1 staged T3C1G1. The average size was 2.94 $\mathrm{cm}$ (range $0.5-9.5 \mathrm{~cm}$ ), the average DOI was $11.4 \%$ with 25 specimens having no myometrial invasion, and there was only 1 specimen with LVSI (the patient with stage T3 disease).

At the time of hysterectomy, 61 patients had a BSO: 51 patients were in the cancer cohort and 24 in the CAH/EIN group, 1 patient had a prior BSO. 4 patients had a bilateral salpingectomy with ovarian preservation, all in the CAH/EIN group. 64 patients had washings done, 22 in the CAH group, 42 of the cancer group. Sixty-six patients had minimally invasive surgery to include vaginal hysterectomy, laparoscopic assisted, and robot assisted hysterectomy. Seven patients in the cancer group had a TAH, 5 patients in the CAH/EIN group had a TAH. All open abdominal surgeries were done for uterine size. Thirtyfour patients had a lymph node dissection (LND) performed at the time of their surgery.

Fifty-five $(61.8 \%)$ hysterectomies were done by the primary author, 5 by other contracted gynecologic oncologists, and the remaining hysterectomies performed by general gynecology. Three restaging surgeries were performed by the primary author, of those one didn't qualify by Mayo criteria but was per patient request after extensive shared decision making.

Per Mayo criteria: 33 (64.1\%) patients should have been staged, mainly by size criteria. Of these, 30 should have been staged by size of tumor alone, as there was either no depth of invasion (DOI) or $<1 / 2$ DOI and pathologic grade was 1 or 2 . Four $(7.84 \%)$ should have been staged by DOI only.

Five of 51 (9.8\%) should have been surgically staged per the French normogram stratified by risk of LN metastasis at $>5 \%$. Three of these did have LND performed at time of index surgery (of note, there was no size criterion for this normogram).

Only 1 patient met both Mayo and the French criteria. This patient, who was predicted to have positive LN by both methods, had her index surgery performed by sub-specialty. Her final stage was T3C1G1 and she had a calculated $13 \%$ risk per the French normogram. A modified Mayo criteria without tumor size was then reviewed. When comparing the modified Mayo criteria without tumor size to the French normogram using the Mann-Whitney $U$ test, the $p=0.6732$ and was not statistically significant.

LND was performed on 34 patients ( $43.5 \%$ of all patients, $66 \%$ of those diagnosed with cancer). Only 1 patient was found to have LN metastasis of the 51 patients with a final cancer diagnosis.

Fifteen patients had hormonal suppression prior to their hysterectomy, most commonly a progestin IUD placed at the time of hysteroscopy D\&C: 10 in the CAH/EIN cohort of whom 5 used an IUD, and 5 in the cancer cohort, of whom 4 used an IUD. This was statistically significant with the two-tailed $\mathrm{p}=0.0033$ (95\% CI: -0.45 to $-0.09, \mathrm{t}=3.0345)$. This difference may represent progestin suppression either stabilizing CAH/EIN or enabling regression of cancer back to $\mathrm{CAH} / \mathrm{EIN}$. All patients are NED at the time of publication.

\section{Discussion}

This review has shown that there continues to be a concordant risk of uterine cancer in those women diagnosed with CAH/EIN. The risk was independently and positively associated with a preoperative pathologic diagnosis of an "at least" or "cannot rule out" cancer comment. The risk was negatively associated with preoperative progestin use. There was no association with medical comorbidities or BMI, means or biopsy, or route of surgery.

Due to this quality improvement review, a meaningful discussion between pathology and gynecologic oncology has ensued. When the preoperative diagnosis contains one of the above comments, we found that the pathologists have seen components of cancer within the specimen, but prevaricated wording so as to not definitively assign the patient a cancer diagnosis. We have then mitigated this equivocation, and made the diagnosis wholly scientific to include: $2 \times 2 \mathrm{~mm}$ of back to back glands, and glandular cytologic atypia identified in the presence of desmoplastic reaction. A surface papillary pattern, mazelike meandering glands and solid non-squamoid areas also suggest an adenocarcinoma. If we could have eliminated the "can't rule out" and associated comments connected with the preoperative biopsy results, $55 \%(28 / 51)$ of patients would have had direct referral to sub-specialty.

In an early GOG (33) study, the authors suggested that a lowrisk group existed in which routine $\mathrm{LN}$ dissection was not needed [13]. However, after FIGO included lymphadenectomy in the surgical 
staging of endometrial cancer in 1988, the procedure was endorsed as a standard surgical treatment by many practice guidelines. Many researchers have attempted to identify a low-risk group to avoid morbidity associated with unnecessary LN dissection. The most wellknown assessment algorithm is the intraoperative decision criteria from the Mayo Clinic. In a review of 815 patients, Mariani et al. indicated that a low-risk group could be defined as patients with: (i) endometrioid subtype; (ii) myometrial invasion of $50 \%$ or less; (iii) histological grade 1-2; (iv) no intraoperative evidence of macroscopic disease., and tumor size; (v) diameter of $<2 \mathrm{~cm}$ [14]. The authors claimed that these patients can be treated optimally with hysterectomy without LND.

Japanese researchers developed an algorithm incorporating serum CA125 and preoperative MRI. Todo et al. reported that patients with none of the following risk factors (to include: elevated CA125 $\geq 70 \mathrm{U} /$ $\mathrm{mL}$ if age $<50$ years; CA1 $25 \geq 28$, if age $\geq 50$ years, a volume index $<25$, serous histology, and grade 3 ) have a $3.6 \%$ risk of pelvic LN metastasis [15]. The authors stated that LND can be omitted in this low risk group. The authors modified their scoring system and identified four risk factors (grade 3 or serous histological subtype, volume index $\geq 36$, magnetic resonance imaging [MRI]-based myometrial invasion $\geq 1 / 2$, and serum CA125 level $(\geq 70 \mathrm{U} / \mathrm{ml}$, if age $<50$ years; $\geq 28$, if age $\geq 50$ years) and found if absent, that, $3.3 \%$ of these low risk patients were found to have LN node metastasis.

Koskas et al. have also developed an algorithm that is predictive of LN metastasis and has been internally and externally validated in France. We compared this normogram with the Mayo criteria which is widely used in the United States. We found the Mayo criteria with size inclusion predisposed $64.7 \%$ of patients to LND and surgically overtreated $62.7 \%$ of patients. When size criteria were removed, $7.8 \%$ of patients were deemed high risk enough for LND. When we compared the Mayo criteria without tumor size to the French normogram, the outcomes were not statistically different. The French criteria is then a functional assessment method for risk stratification of LND in uterine cancer patients as well.

Regarding final outcomes of those patients who had a cancer diagnosed on final pathology: $8-10 \%$ of patients would have qualified for LND using either a modified Mayo criteria (eliminating tumor size) or the French algorithm. Of those $10 \%$, only 1 patient had LN metastasis. No patient was adversely affected by the surgeon who performed their index surgery. Regarding the patient who had the LN involvement, she had surgery with sub-specialty because she was identified high risk by preoperative algorithms including an elevated ca125 drawn because of imaging identification of a concurrent adnexal mass.

In conclusion, denoting uterine precancer as EIN instead of CAH to accommodate international standard pathology terminology, and eliminating comments to include "possible, or cannot rule out" cancer, may help direct patients to appropriate specialty vs sub-specialty care. For those patients with a diagnosis of CAH/EIN on preoperative biopsy but discordant with final cancer pathology, gynecologists in this study did not adversely affect patient outcomes. Normograms for LN metastasis prediction were applied to include the Mayo and French criteria. A modified Mayo criteria and the French criteria provided retrospective accuracy in assessment of $\mathrm{LN}$ metastasis.

\section{References}

1. O'Dowd MJ, Philipp EE. The history of Obstetrics and Gynaecology. 1st ed. New York: Parthenon Publishing Group; 1994. Cancer of the uterus; pp. 571-80.

2. Kurman RJ, Kaminski PF, Norris HJ (1985) The behavior of endometrial hyperplasia. A long-term study of "untreated" hyperplasia in 170 patients. Cancer 56: 403-412. [Crossref]
3. ACOG Committee opinion: Number 631, May 2015 Endometrial Intraepithelial Neoplasia

4. Baak JP, Mutter GL, Robboy S, van Diest PJ, Uyterlinde AM, et al. (2005) The molecular genetics and morphometry-based endometrial intraepithelial neoplasia classification system predicts disease progression in endometrial hyperplasia more accurately than the 1994 World Health Organization classification system. Cancer 103: 2304-2312. [Crossref]

5. Mutter GL (2000) Endometrial intraepithelial neoplasia (EIN): will it bring order to chaos? The Endometrial Collaborative Group. Gynecol Oncol 76: 287-290. [Crossref]

6. Trimble CL, Kauderer J, Zaino R, Silverberg S, Lim PC, et al. Concurrent endometrial carcinoma in women with a biopsy diagnosis of atypical endometrial hyperplasia. A Gynecologic Oncology Group study. Cancer 106: 812-819. [Crossref]

7. Bokhman JV (1983) Two pathogenetic types of endometrial carcinoma. Gynecol Oncol 15: 10-17. [Crossref]

8. Douglas A. Levine, DA, The Cancer Genome Atlas Research Network (2013) Integrated genomic characterization of endometrial carcinoma. Nature 497: 67-73.

9. AlHilli MM, Podratz KC, Dowdy SC, Bakkum-Gamez JN, Weaver AL, et al. Riskscoring system for the individualized prediction of lymphatic dissemination in patients with endometrioid endometrial cancer. Gynecol Oncol 131: 103-108. [Crossref]

10. Bendifallah S, Genin AS, Naoura I, Chabbert Buffet N, Clavel Chapelon F (2012) A nomogram for predicting lymph node metastasis of presumed stage I and II endometrial cancer. Am J Obstet Gynecol 207: 197. [Crossref]

11. Koskas M, Fournier M, Vanderstraeten A, Walker F, Timmerman D, et al. (2016) Evaluation of models to predict lymph node metastasis in endometrial cancer: A multicentre study. Eur J Cancer 61: 52-60. [Crossref]

12. Benedetti Panici P, Basile S, Maneschi F, Alberto Lissoni A, Signorelli M, et al (2008) Systematic pelvic lymphadenectomy vs. no lymphadenectomy in early-stage endometrial carcinoma: randomized clinical trial. J Natl Cancer Inst 100: 1707-1716. [Crossref]

13. Creasman WT, Morrow CP, Bundy BN, Homesley HD, et al. Surgical pathologic spread patterns of endometrial cancer. A Gynecologic Oncology Group Study. Cancer 60: 2035-2041. [Crossref]

14. Mariani A, Dowdy SC, Cliby WA, Gostout BS, Jones MB, et al. Prospective assessment of lymphatic dissemination in endometrial cancer: A paradigm shift in surgical staging. Gynecol Oncol 109: 11-28. [Crossref]

15. Kang S, Todo Y, Odagiri T, Mitamura T, Watari H, et al. (2013) A low-risk group for lymph node metastasis is accurately identified by Korean gynecologic oncology group criteria in two Japanese cohorts with endometrial cancer. Gynecol Oncol 129: 33-37. [Crossref]

Copyright: (C)2018 Benoit MF. This is an open-access article distributed under the terms of the Creative Commons Attribution License, which permits unrestricted use, distribution, and reproduction in any medium, provided the original author and source are credited. 